\title{
X-linked intellectual disability-cubitus valgus-dysmorphism syndrome
}

INSERM

\section{Source}

INSERM. (1999). Orphanet: an online rare disease and orphan drug data base. $\underline{x \text {-linked }}$ intellectual disability-cubitus valgus-dysmorphism syndrome. ORPHA:85280

X-linked intellectual disability-cubitus valgus-dysmorphism syndrome is characterised by moderate intellectual deficit, marked cubitus valgus, mild microcephaly, a short philtrum, deep-set eyes, downslanting palpebral fissures and multiple nevi. Less than ten individuals have been described so far. Transmission is thought to be $\mathrm{X}$-linked recessive. 